\title{
On the use of the periodicity condition in cross-flow tube
}

\author{
Boumedienne Beladjine ${ }^{1}$, Mohammed Aounallah ${ }^{2}$, a, Mustapha Belkadi ${ }^{2}$, Lahouari Aadjlout ${ }^{2}$, Omar Imine $^{3}$ \\ ${ }^{1}$ Laboratory of Energy and Naval Propulsion, University of Sciences and Technology of Oran-Mohamed Boudiaf, \\ P. O. 1505 El-Mnaouar Oran Algeria. \\ ${ }^{2}$ Laboratory of Aero-Hydrodynamics Naval, University of Sciences and Technology of Oran-Mohamed Boudiaf, \\ P. O. 1505 El-Mnaouar Oran Algeria. \\ ${ }^{3}$ Laboratory of Aeronautics and Propulsive Systems, University of Sciences and Technology of Oran-Mohamed \\ Boudiaf, P. O. 1505 El-Mnaouar Oran Algeria.
}

\begin{abstract}
This paper presents the results of measurements and numerical predictions of turbulent cross-flow through an in-line $7 \times 7$ bundle configuration with a constant transverse and longitudinal pitch-to-diameter ratio of 1.44. The experiments are conducted to measure the pressure around tubes, using DPS differential pressure scanner with air flow, in square channel at a Reynolds number of 35000 based on the gap velocity and the tube diameter. The commercial ANSYS FLUENT is used to solve the unsteady Reynolds-Averaged Navier-Stokes (RANS) equations. The primary aim of the present study is to search for a turbulent model that could serve as an engineering design tool at a relatively low computational cost. The performances of the Spalart-Allmaras, the RNG k- $\varepsilon$, the Shear Stress Transport k- $\omega$ and the second moment closure RSM models are evaluated by comparing their simulation results against experimental data. The second objective is to verify the validity of the periodicity assumption taken account in the most previous numerical works by considering the filled bundle geometry. The CFD results show that the Spalart-Allmaras model on the fine mesh are comparable to the experiments while the periodicity statement did not produce consistently the flow behavior in the $7 \times 7$ tube bundle configuration.
\end{abstract}

\section{Introduction}

Flow in tube bundles have many important industrial applications and have been studied both experimentally and through numerical simulations. The ability to correctly predict complex turbulent flows is fundamental to the design of many fluid engineering systems. In spite of the great number of scientific contributions related to the cross-flow turbulent tube bundle, few numerical works were carried out to investigate the dynamic filed in the entire tube bundle. The majority of the studies consider only a partial domain enclosed with periodic boundary conditions.

Launder and Massey [1] obtain the flow field for a staggered tube bundle at Reynolds number up to 1000 applying periodic boundary conditions under the assumption of fully developed flow. The authors demonstrate the same pattern of the velocity filed in every spatial period and propose an arithmetical law for the pressure difference. Simonin and Barcouda [2] perform LDA measurements and numerical studies of the turbulent cross-flow in a tube bundle with different pitch ratio; they visualize a small recirculation zone in the wakes of tubes with fairly high turbulence intensity values and highest length of the recirculation zone behind the first row. Numerically, they report a good agreement for the mean velocities and a significant discrepancy in the prediction of the turbulent kinetic energy with the k- $\varepsilon$ model. Sebag et al. [3] use non-standard coefficients for Reynolds Stress Transport Models (RSTM) and paradoxically the standard $\mathrm{k}-\varepsilon$ model returns reasonable predictions of mean velocities and global level of turbulent kinetic energy. They note that is purely by chance thanks to the erroneous overproduction of kinetic energy on the impinging side of the tubes while its maximum and minimum locations are wrong. Meyer [4] uses the LDA technique to measure $2 \mathrm{D}$ mean velocities and Reynolds stresses in two successive rows in the middle of tube bundle. The measurements are compared with the numerical predictions using the standard $\mathrm{k}-\varepsilon$ model and the second moment closure, both models give poor predictions of the Reynolds stresses. Zukauskas and Ulinskas [5] present some experimental results on the characteristics of the flow and the heat transfer around a single tube and cross-flow tube bundle. They establish that, in the sub-critical flow regime, the pressure drop in a tube bundle decreases with Reynolds number but beyond a critical Reynolds number it increases. They also report

\footnotetext{
${ }^{\mathrm{a}}$ Corresponding author: aounallah_2000@yahoo.fr
} 
correlations for the total pressure drop and heat transfer coefficients for a wide range of Reynolds numbers and several tube bundle configurations. Rollet-Miet et al. [6] use the LES technique and the $\mathrm{k}-\varepsilon$ model to predict the turbulent flow as produced by the experiment of Simonin and Barcouda [2]. Both LES and $\mathrm{k}-\varepsilon$ models calculate the mean velocity profiles reasonably well while $\mathrm{k}-\varepsilon$ model gives poor predictions of the Reynolds stresses in the wake region inconsistency to the LES results. Benhamadouche et al. [7] compute the cross-flow in a staggered tube bundle with LES and a transient Reynolds Stress Transport Models (RSTM) in 2D and 3D configurations with two levels of grid refinement. It is shown that the LES results on the fine mesh are comparable to the DNS and the experiment and reasonable agreement is still achieved with a coarse mesh. The RSTM produces also satisfactory results in 3D but showed no advantage over the LES when the grid is coarse. The 2D RSTM, which produced strong vortex shedding, is found to be physically unreasonable. Moulinec et al. [8] use the DNS technique for a Reynolds number of 6000 to predict the flow field. The NavierStokes equations are discretized on a staggered grid with the finite volume approach. Overall, they report that their predictions are in good agreement with the experiment. Paul et al. [9] use the low-Reynolds number $\mathrm{k}-\varepsilon$, the $\mathrm{k}-\omega$ and the $\varepsilon$-based second moment closure models to predict the flow in the typical section of the experiment of Simonin and Barcouda [2]. They report that the $k-\varepsilon$ and the $\mathrm{k}-\omega$ models give better predictions of the mean flow in the region just before and after the tube than the second moment closure (LRR-IP) model. Moreover, this latter does not produce consistently good predictions of the Reynolds stresses. Benhamadouche et al. [10] examine the influence of the pitch ratio $(P / D=1.44$ and $P / D=1.75)$ for a cross-flow $3 \mathrm{D}$ square in-line tube bundle by means of LES with periodic boundary conditions and using the in-house EDF code Saturne and $C D$-Adapco code STAR CCM. It is shown that a high accuracy is needed for pressure gradient calculation to obtain a physical solution. Drag and lift coefficients have reasonable values, but their $r m s$ values are very sensitive to the extrusion length. Mizushima and Suehiro [11] investigate the transition of flow past only two tubes in tandem arrangement, which can be considered as a very simple model of tube bundle, and found a hysteresis in the amplitude of velocity in the oscillatory flow. They explain that the hysteresis comes from a bending of solution branch due to non-linear interactions of the wake with the downstream tube, which leads to two stable oscillatory solution branches connected to each other through an unstable solution branch. Paul et al. [12] achieve measurements in a staggered tube bundle with fixed pitch ratio at sub-critical Reynolds number using PIV technique. Their results show that the flow exhibits strong Reynolds number dependence in the developing region, however no significant effect is observed in the spatially periodic region. Imran [13] studies the turbulent flow through square in-line arrays using various RANS and LES simulations. Several flow parameters such as velocity profiles, pressure distributions, instantaneous and RMS lift and drag coefficients and Reynolds stresses are compared to the available experimental data. The author found an unexpected asymmetric behavior with flow switching directions. Yahiaoui et al. [14] investigate experimentally the turbulent flow through an in-line tube bundle formed by $7 \times 7$ tubes arranged with a constant transverse and longitudinal pitch ratio of 1.44 . The pressure distribution on the tube surface indicates the stagnation pressure region located somewhere around $45^{\circ}$ similarly to the LES results of Imran [13].

The first aim of the present study is to perform detailed assessment of the ability of the commercial CFD code, ANSYS Fluent, together with four different turbulence models in their unsteady state formulations, Spalart- Allmaras, RNG k- $-\varepsilon$, SST k- $\omega$, and RSM second moment closure, to reproduce the mean flow in the inline tube bundle. The good agreement of the numerical results with the experimental measurements associated with a relatively low computational cost referee the better model. As mentioned previously, most of the numerical investigations have treated this problem with the periodic boundary conditions assumption to reduce the grid size and consequently the CPU time. Here, the entire tube bundle configuration formed by the whole 49 tubes is considered to verify the validity of the periodicity condition. The turbulent flow through the tube bundle is assumed as a two-dimensional case although it is generally characterized by significant 3D flow structures and highly fluctuating wake regions with vortex shedding from the time when Yahiaoui et al. [14] confirm the same trend of the pressure coefficient, the drag and lift coefficients distributions for different transversal positions along the tubes.

\section{Experimental setup and measurement procedure}

The experiments are carried out in a closed-circuit air tunnel with a test section of $460 \mathrm{~mm}$ wide, $460 \mathrm{~mm}$ deep and $1000 \mathrm{~mm}$ long. It is driven by an A.C motor and axial flow fan that forces air around the circuit and produces a maximum velocity of $60 \mathrm{~m} / \mathrm{s}$. The test section is made with a clear Plexiglas to facilitate visualization. The bundle contains 7 rows and 7 arrays of PVC tubes of outer diameter of $40 \mathrm{~mm}$ arranged in square in-line arrays. Half tubes are also fixed along the top and bottom walls of the test model to simulate infinite tube bundle and minimize the wall boundary layers. The pitch ratio is respected in both direction; longitudinal and transverse $(P / D=T / D=1.44)$. The deep-to-diameter ratio is equal to 11.5 , larger than 7.2 used by Balabani et al. [15], who consider measurements at the mid-span to be free from the end effects. The air with an approach velocity, $U_{\text {inf }}=$ $3.13 \mathrm{~m} / \mathrm{s}$ enters to the test section uniformly giving a Reynolds number of 35000 based on the tube diameter and the gap bulk velocity $U_{\text {gap }}=10.25 \mathrm{~m} / \mathrm{s}$. Figure 1 shows the cross-sectional view of the tube bundle model with the definition of geometrical parameters. For convention, each tube is coded by a number; Tube 23 is located in the second line from the bottom and the third column from the right. The angle measurement is similar to counter clockwise direction and starts from the inlet free stream direction. 


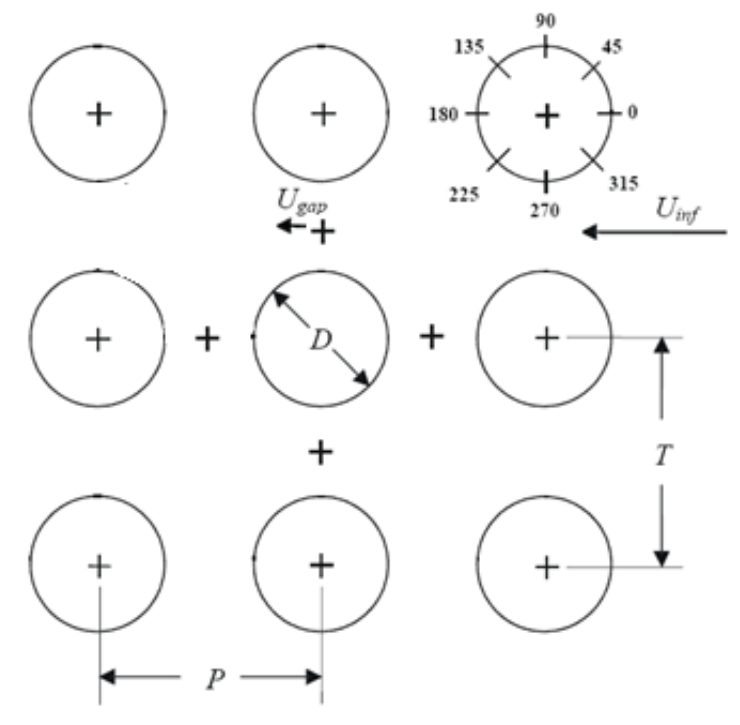

Figure 1. Cross-flow through the in-line square tube bundle.

The pressure measurements are done using the TE44 DPS differential pressure scanner. The latter pressure scanning box allowed sequential selection of up to 20 pressure tapings. The display unit links to a computer, loaded with DATASLIM software for data analysis and logging of results. Figure 2 shows the pressure coefficient measurement deviation for the pivot tube 44 . It can be seen that the uncertainty analysis of the present measurement is insignificant since the uncertainly in the mean pressure at $95 \%$ confidence level is estimated to be $\pm 4 \%$ and the standard deviation is only of the order of $1.6 \%$.

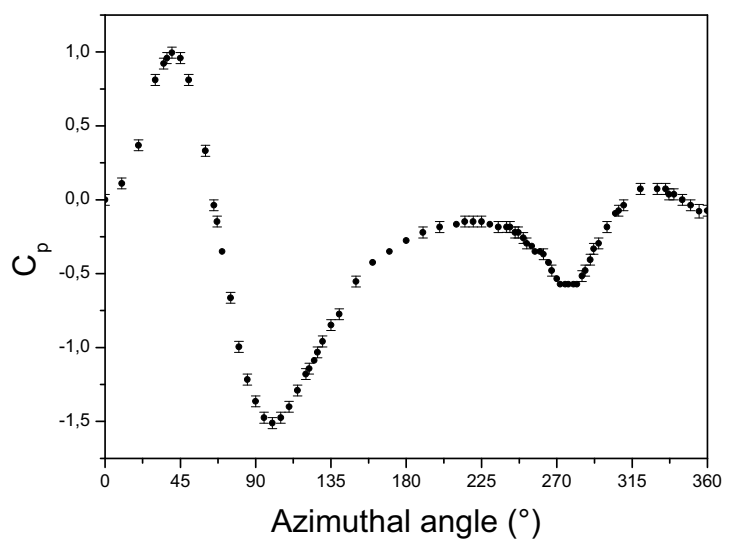

Figure 2. Pressure coefficient measurement deviation for the tube 44 .

\section{Numerical modeling}

The geometry of the 2D domain representing the typical region in the in-line bundle with the tube diameter $D$, the transverse pitch $P$ and the longitudinal pitch $T$ is shown in Figure 1. The same number of tubes in the experimental investigation is considered for the simulations. The two dimensional assumption is validated by Paul et al. [12] who clearly demonstrate the $2 \mathrm{D}$ flow in the mid-plane of the channel. The flow is assumed to be unsteady and turbulent. The incompressible fluid is Newtonian with constant density $\rho$ and dynamic viscosity $\mu$. The body force due to buoyancy is neglected. The equations governing the development of the flow through the tube bundle are as follows:

$$
\begin{gathered}
\frac{\partial U_{j}}{\partial x_{j}}=0 \\
\frac{\partial U_{j}}{\partial t}+\frac{\partial}{\partial x_{j}}\left(\rho U_{j} U_{i}\right)=-\frac{\partial P}{\partial x_{i}}+\frac{\partial}{\partial x_{j}}\left(\mu \frac{\partial U_{i}}{\partial x_{j}}-\rho \overline{u_{i} u_{j}}\right)
\end{gathered}
$$

where $\overline{u_{i} u_{j}}$ is the Reynolds stress tensor. The mean flow pressure is $P$ and the mean velocity $U_{i}$. The boundary conditions for the computational domain are defined as follows. At the inlet, an average upstream value of the mean velocity, $U_{i n f}=3.13 \mathrm{~m} / \mathrm{s}$ fixed from measurements and a turbulence intensity of $4 \%$ are specified, the turbulent quantities are estimated using the turbulence intensity and the hydraulic diameter of tube. The no-slip boundary condition is prescribed on the tube surfaces, the bottom and top walls. At the exit, a pressure-outlet condition is specified with a reference value of zero. Several turbulence models are used; the one equation model developed by Spalart-Allmaras [16], the twoequation of renormalization group $\mathrm{k}-\varepsilon$ developed by Yakhot and Orszag [17], the shear stress transport (SST) k- $\omega$ model developed by Menter [18] and the second moment closure Reynolds Stress Model developed by Launder et al. [19].

\section{Numerical Method}

The coupled conservation equations are resolved numerically using the commercial code ANSYS Fluent 6.3.26 with the segregated solver and the finite volume method approach of Patankar [20]. The partial differential equations are discretized on a grid where all the variables are collocated. The second-order upwind scheme is used in the momentum and the turbulence transport discretization while the standard scheme is employed for the pressure equation. The SIMPLE algorithm is used to deal with the pressure-velocity coupling between the momentum and the continuity equations. The computations are done with double precision and the solution is achieved when all residuals of the discretized equations fall down $10^{-5}$. The relaxation parameters are adjusted for each simulation in order to accelerate convergence. The computational domain is filled with a non-uniform hybrid grid with a very fine spacing near the tube walls, as needed for accurately resolving the steep gradients in the thin boundary layer. Mesh-independence tests are performed to investigate the influence of grid refinement on the solution. Figure 3 shows a partial view of the computational domain with the coarse and the fine mesh. 


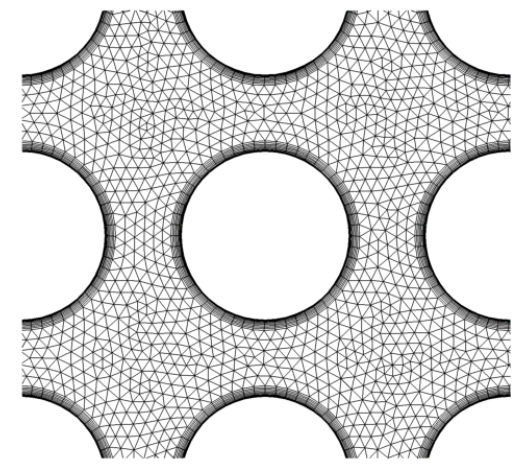

a) Coarse mesh

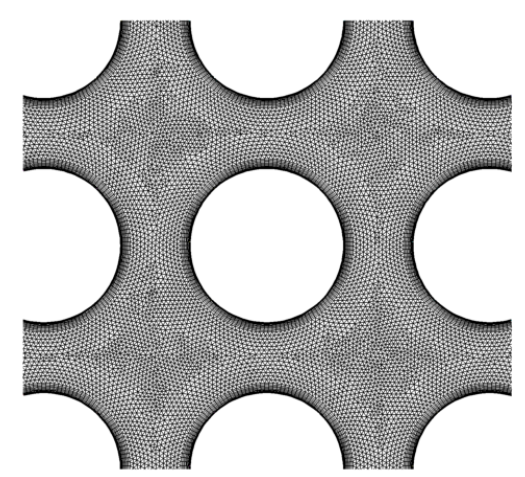

b) Fine mesh c)

Figure 3. A partial view of the computational domain.

\section{Validations}

Mesh-independence tests are performed to investigate the influence of grid refinement on the solution. Table 1 provides some details of the three grids tested including the total number of nodes and cells, the number of nodes along the longitudinal, the transversal directions and on the tube surface respectively. The boundary layer region around tubes is formed by 8 layers with an expansion factor of 1.1. Many numerical tests have been carried out in this section to choose the suitable grid that provides the closest results to the experimental measurement.

Table1. Grids details used for mesh-independence tests.

\begin{tabular}{lrrrrr}
\hline Grid & Nodes & Cells & $N_{x}$ & $N_{y}$ & $N_{\text {tube }}$ \\
& & & & & \\
\hline coarse & 51060 & 78512 & 290 & 96 & 52 \\
medium & 159318 & 271312 & 528 & 192 & 104 \\
fine & 236377 & 413508 & 751 & 256 & 130 \\
\hline
\end{tabular}

Figure 4 shows the computed pressure coefficient distribution around the tube 33 (row: 3, column: 3 ) in terms of azimuthal angle. In overall, the pressure curve shows an asymmetric distribution with a fairly high pressure region on the windward side and a low pressure region on the leeward side. This asymmetry is created from the deflection of the flow through array. In this section, only the grid independence for the SpalartAllmaras model is presented. As will be seen later, this simple one-equation model could be a very promising tool for numerical simulation of complex turbulent flows through the tube bundle compared to the two-equation models assumed eddy-viscosity and the second moment closure model. Qualitatively, all the grids tested give the same trend of the pressure coefficient. The fine mesh gives more neighboring value to the experimental results [14] and the LES of Imran [13], particularly in the range between $120^{\circ}-240^{\circ}$, while the deficit of the coarse and the medium grids is obviously located among $75^{\circ}$ and $180^{\circ}$. Surprisingly, the coarse grid surmounts the others grids by the good capture of the high pressure around the stagnation point behind the angle of $45^{\circ}$ where the medium mesh underestimates dramatically the pressure distribution. It is clearly seen that the experimental measurement forecasts a constant pressure distribution from the angle $240^{\circ}$ until $300^{\circ}$ in opposition to the numerical predictions which predict a decrease then an increase. From the two thirds of the tube, all the grids tried are unable to predict the physical behavior of the pressure distribution even the LES. In overall and on the complete circumference of the tube, the fine mesh can be kept as the suitable one that reproduces neighboring pressure distribution to the experiment results.

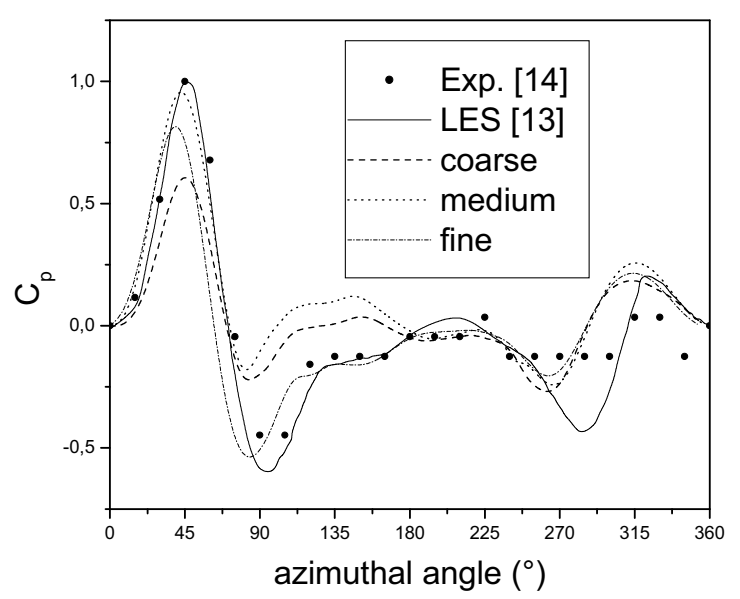

Figure 4. Grid independence tests of the Spalart-Allmaras model.

Figure 5 illustrates the ability of the turbulence models used to resolve the flow characteristics using the fine grid and namely the pressure distribution along the tube 33 surface. Once again, the asymmetric pressure distribution due to the flow direction switching is observed. The stagnation point is at an angle of 45 degrees, as expected in the literature. Lam and Fang [21] found the location of the high pressure in the angle range of 20-60 degrees. The adverse pressure region on the leeward side which corresponds to an angle range from 80 to 300 degrees with a sharp dip at 90 degrees, leads to an almost no zero net lift force. In comparison with the experimental data of Yahiaoui [14] and the numerical LES computation conducted by Imran [13], the general tendency of the pressure coefficient is set up by all the 
RANS models tested with some quantitative discrepancies. As common remarks, all the turbulence models underestimate the pressure peak at the stagnation point and none can establish the null pressure gradient in the angle range between $240^{\circ}$ and $300^{\circ}$ even the LES. A strong decrease and increase of pressure is observed in this area. Inconsistently, the simple one equation model; Spalarat-Allmaras agree enough well with experiment in spite of the advance position of the stagnation point. The RNG k- $\varepsilon$ model is reasonably good but it dramatically underestimates the value of the stagnation point and overestimates the pressure at the angle of $90^{\circ}$. Obviously, the shear stress model k- $\omega$ SST seems to be somewhat failed due to the underestimation of the pressure coefficient at the angle of $45^{\circ}$ and exclusively to the over assessment of the pressure in the range $90^{\circ}-180^{\circ}$. As for the RSM model, it can be refereed less good than the other models. This latter underestimates the pressure coefficient on the majority surface of the tube and also over predicts the pressure at the angle $90^{\circ}$. With that, it should not forget the warrant of the additional computational expense. Finally, it is important to note the poor quality of the numerical models tested even the LES in the region $240^{\circ}-300^{\circ}$, where the experimental measurements attest a null pressure gradient.

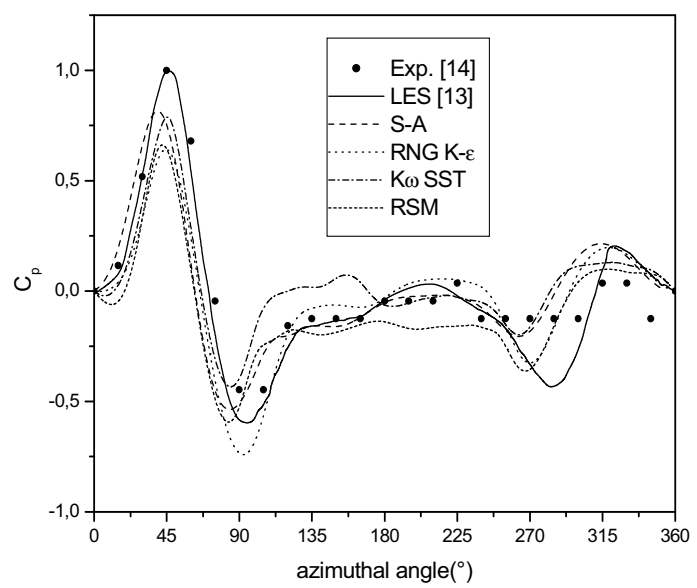

Figure 5. Pressure coefficient distributions along the tube 33.

\section{Results and discussion}

Figure 6 shows the streamlines across the tube bundle obtained with the Spalart-Allmaras model discretized on the fine grid at $\mathrm{t}=15 \mathrm{~s}$. This figure ascertains the nonresemblance of the flow behavior in the space for the two different times, the flow pattern around any tube is not similar to it adjacent. It is apparent from both contours, that the periodicity boundary condition is not valid to simulate the cross-flow in tube bundle. The random and the chaotic characters of the flow are obviously seen on the full computational domain. Following the flow direction, the streamlines are nearly horizontal in the passages trough the tubes. Different vortices with different size and opposite in rotational direction are shaped backstream of some tubes. It is also interesting to note that the flow pattern in the first row is qualitatively similar to that an isolated single cross-flow. The flow becomes oscillatory downstream the last array and large vortices with different rotation direction tend to be formed. After the wake region, the streamlines becomes regular and horizontal.



Figure 6. Flow pattern in the tube bundle.

In order to check the validity of the periodicity condition, a particular attention is allocated to the pressure coefficient predictions of the central horizontal line and the middle vertical column tubes. Igarashi [22] classify the flow pattern into three types according to the maximum value of the pressure coefficient and its positions. The three situations are designated as $W, R$ and $J$ hereinafter, respectively. The situation without reattachment of the shear layer, in which the $C_{p}$ value has no maximum, belongs to the first type. The situation with reattachment of the shear layer, in which the $C_{p}$ value has a maximum, belongs to the second type. The situation of rolling up of the shear layer in front region of the cylinder, in which the $C_{p}$ value has maximum at $0^{\circ}$ angle, belongs to the third type.

Figure 7 reveals the pressure distribution comparison in the fourth vertical column. As can be seen, the same trend of the pressure coefficient along the border tubes is established by both experimental measurements and numerical predictions. The reattachment of the shear layer is experimentally located in the range $40^{\circ}-50^{\circ}$ and fairly found advanced with the numerical computation $\left(25^{\circ}-35^{\circ}\right)$. This remark is also valid for the separation of the shear layer indicated by the lower pressure coefficient around the angle $100^{\circ}$. The major quantitative discrepancy of the numerical approach compared to the experiment is well realized in the angle range of $330^{\circ}$ $360^{\circ}$, it represents a second reattachment. Unless this latter statement, it can be concluded that the flow shows unexpected symmetric behavior in the transverse direction. 

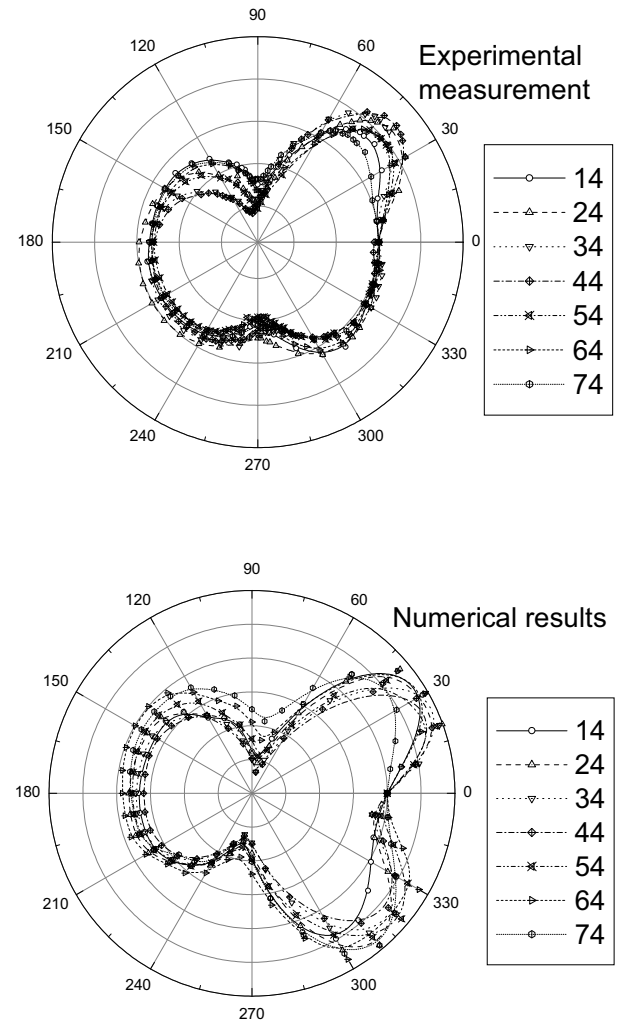

Figure 7. Pressure coefficient distribution in the $4^{\text {th }}$ column.

Following the horizontal middle row and contrary to the vertical array, notable qualitative and quantitative differences exist between both methodologies as can be shown in figure 8 with the only similarity of the first tube, where the pressure action appears fairly symmetric and resemble to an isolated cylinder. Numerically, the profiles of the successive tubes are almost the same; two stagnation points indicating the reattachment of the shear layer are predicted around $25^{\circ}$ and $315^{\circ}$, while experimental measurement explored switched different pressure distributions. The measurements show that the cylinders are more solicited close to the $45^{\circ}$ angle with different intensity except tube 43 which has it stagnation point at $325^{\circ}$. The flow shows unexpected asymmetric behavior with flow switching directions. So, it can be conclude that no periodic pattern occurs in this direction.

\section{Conclusion}

In the present study, Experimental and numerical investigations of the turbulent flow through an in-line $7 \times 7$ bundle configuration with a constant transverse and longitudinal pitch-to-diameter ratio of 1.44 were carried out. The investigation covers the validity of the periodic boundary conditions with the appropriate turbulence model. The DPS differential pressure scanner technique is used to perform detailed measurements. Turbulence is
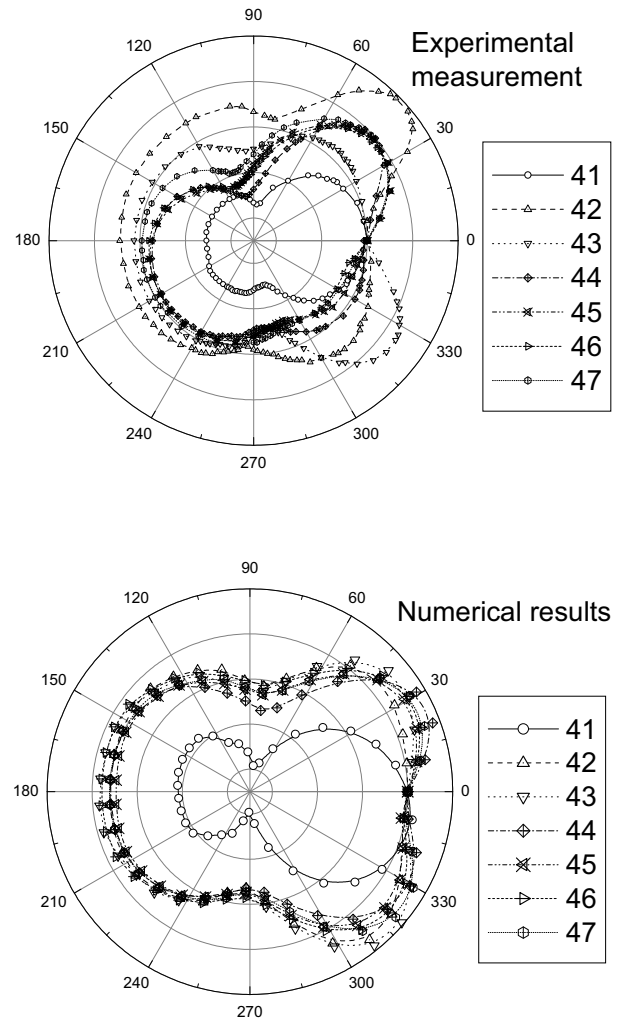

Figure 8. Pressure coefficient distribution in the $4^{\text {th }}$ row.

modelled and tested by four models to resort the best one that expresses the closest pressure distribution to the experimental data.

The numerical results confirm that with carefully designed grid resolution and distribution, the SpalartAllmaras model can predict rather well the characteristics of turbulent flow past the tube bundle in spite of the advance location of the stagnation point. The flow pattern obtained numerically inside the full computational domain is characterized by a totally variance in space sanctioning the use the periodicity boundary condition. The number and the size of the vortices formed downstream tubes are markedly unalike and disappear altogether the last tube. It is confirmed by the significant difference of the pressure distribution curves on the horizontally and vertically central tubes. The results reveal also the instantaneous pressure and velocity magnitude fields and pressure which describe the complex flow characteristics in tube bundles. This implies that further measurements and numerical tests should be conducted in the future to more comprehend such complex flow. 


\section{References}

1. B. E. Launder and T. H. Massey, J. Heat Transfer, vol. 100, (1978)

2. O. Simonin and M. Barcouda, Fourth International Symposium on Applications of Laser Anemometry to Fluid Mechanics. Lisbon, Portugal, (1988)

3. S. Sebag, V. Maupu, D. Laurence, TSF8, (1991)

4. K.E. Meyer, Experimental and numerical investigation of turbulent flow and heat transfer in staggered tube Bundles. $\mathrm{PhD}$ thesis, Technical University of Denmark, (1994)

5. A. Zukauskas and R. Ulinskas, Banks of plain and finned tubes. In: Hewitt, G.F. (Ed.), Heat Exchanger Design Handbook. HEDH, New York, 2.2.4-12.2.4-17, (1998)

6. P. Rollet-Miet, D. Laurence, J. Ferziger, Int. J. Heat Fluid Flow 20, (1999)

7. S. Benhamadouche and D. Laurence, Int. J. Heat Fluid Flow 24, (2003)

8. C. Moulinec, M. J. B. M. Pourquie, B. J. Boersma, F. T. M. Nieuwstadt, Int. J. Comput. Fluid Dyn. 18 (1), (2004)

9. S. S. Paul, S. J. Ormiston, M. F. Tachie, Proceedings of the $12^{\text {th }}$ Annual Conference of the CFD Society of Canada, Heat Transfer 1, Ottawa, Canada, (2004)

10. S. Benhamadouche, D. Laurence, N. Jarrin, I. Afgan, C. Moulinec, NURETH-11(Nuclear Reactor Thermal-Hyd.), (2005)
11. J. Mizushima, N. Suehiro, Phys. Fluids 17, (2005)

12. S. S. Paul, M. F. Tachie, S. J. Ormiston, Int. J. Heat Fluid Flow 28, (2007)

13. I. Afgan, Large Eddy Simulation of cylindrical Bodies incorporating unstructured finite volume mesh, PhD thesis, University of Manchester, (2007)

14. T. Yahiaoui, L. Adjlout, O. Imine, O. Imine, Mechanika, (2010)

15. S. Balabani, M. Yianneskis, Proc. IMechE Part C, J. Mech. Eng. Sci. 210, (1996)

16. P. Spalart and S. Allmaras, Technical Report AIAA92-0439, American Institute of Aeronautics and Astronautics, (1992)

17. V. Yakhot and S. A. Orszag, Journal of Scientific Computing, 1(1),(1986)

18. F. R. Menter, AIAA J. 32 (8), (1994)

19. B. E. Launder, T. H. Massey, Trans. ASME J. Heat Transfer 100 (4), (1978)

20. S.V. Patankar, Numerical Heat Transfer and Fluid Flow, Hemisphere (McGraw-Hill), New York, (1980)

21. K. Lam, X. Fang, J. of Fluids and Structures, 9. (1995)

22. T. Igarashi, Bulletin of JSME, Vol.29, $\mathrm{N}^{\circ} 249$, (1988) 